\title{
Scope of Audiology in Space Research: A Letter to the Editor
}

\author{
Abishek Umashankar ${ }^{1} \cdot$ Prashanth Prabhu $^{1}$ (D)
}

Received: 3 February 2021 / Accepted: 29 March 2021/Published online: 1 April 2021

(C) Association of Otolaryngologists of India 2021

Astronauts in space are vulnerable to hearing loss due to the travel in the space shuttle and the microgravity environment present in space. The vulnerability to damage in hearing brings in the need for hearing conservation in space flight. Audiologists have played a vital role in hearing conservation for astronauts for the past 20 years in various space centers worldwide. In Houston, Texas, NASA's Johnson space center contains a specialized audiology and hearing conservation clinic to monitor the astronauts hearing sensitivity by performing baseline and an on-orbit hearing assessment $[1,2]$.

NASA encourages AuD (Doctor of Audiology) students to join externships in their space stations and help them explore the hearing conservation techniques used with the help of a multidisciplinary team from acoustic engineers to other health workers. One effective hearing conservation technique is working out solutions to reduce noise exposure and audiologists and other acoustic engineers in establishing a noise hazard inventory. A space audiologist's job is to undergo an On-Orbit Hearing assessment that monitors the crew members' hearing function while they are on board in the spacecraft for a longer duration time. The audiologist must obtain the data and compare the conventional audiometric results between preflight and postflight $[1,2]$.

Prashanth Prabhu

prashanth.audio@gmail.com

Abishek Umashankar

umashankarabishek@gmail.com

1 Department of Audiology All India Institute of Speech and Hearing Mysuru, Naimisham Campus, Road No.3 TK Layout, Manasagangotri, Mysuru, Karnataka, 570006, India
The audiology scope also extends to vestibular assessment and management, and audiologists can play a vital role in managing the balance-related issues in astronauts as they are exposed to zero gravity in space. NASA has sophisticated technology in assessing and treating balancerelated disorders and training astronauts to overcome balance-related problems in zero gravity. Apart from these, experimental research is performed by audiologists in fulfilling the future needs for hearing and balance in space. It may be difficult for some professionals to hire themselves as Audiologists in space agencies directly. However, the transition from airforce can make it easier for Audiologists to accustom to space research as there is a commonality of working in an aeronautical setup. Audiologists are currently hired in the acoustic sub-working group in agencies like NASA, the Russian space agency, the European space agency, and the Canadian space agency. Although the audiologist's scope does extend to space research, it may not be widely known to many working professionals [1-3].

In India, the Indian Space Research Organization (ISRO) has successfully launched various space missions like the Chandrayaan and Mangalyaan to the Moon and Mars [4]. In the upcoming mission, ISRO plans to send at least four astronauts to the moon in the year 2022 [5]. Primarily the astronauts would require a vestibular training and hearing conservation program before space travel. With India having Otorhinolaryngologists and Audiologists specialized in vestibular management and rehabilitation, these professionals' scope would extend to working in the ISRO. Like NASA, additional internship programs can be provided to speech and hearing students, which can enlighten their knowledge of acoustics and hearing in space. 


\section{References}

1. https://roundupreads.jsc.nasa.gov/mobi.aspx?pageid=1392

2. Dicken T (2012) Data Acquisition, Management, and Analysis in Support of the Audiology and Hearing Conservation and the Orbital Debris Program Office

3. Rowden-Racette K (2010) Winding Path Leads Audiologist to NASA: Retired Air Force Clinician Implements Medical Policies for Space Crews. The ASHA Leader 15(8):30-30
4. Mahajan S (2014) ISRO's Mars Mission. Economic and Political Weekly, 10-12

5. Priyadarshini S (2019) India's journey to the red planet. Nature 573(7773):194-196

Publisher's Note Springer Nature remains neutral with regard to jurisdictional claims in published maps and institutional affiliations. 